\title{
The Durability of an Intensive, Structured Education- Based Rehabilitation Protocol for Best Insulin Injection Practice: The ISTERP-2 Study
}

\author{
Sandro Gentile (D) - Giuseppina Guarino • Teresa Della Corte • \\ Giampiero Marino · Ersilia Satta • Maria Pasquarella • Carmine Romano • \\ Carmelo Alfrone · Laura Giordano · Fabrizio Loiacono • \\ Maurizio Capace · Rossella Lamberti · Felice Strollo on behalf of AMD-OSDI Study Group on Injection Technique
}

Received: June 3, 2021 / Accepted: June 25, 2021 / Published online: August 12, 2021

(C) The Author(s) 2021

\section{ABSTRACT}

Introduction: Studies on the durability of an intensive, structured education protocol on best insulin injection practice are missing for people with type 2 diabetes mellitus (T2DM). The aim of this study was to assess the durability of an intensive, structured education-based rehabilitation protocol on best insulin injection practice in well-trained subjects from our previous intensive, multimedia intervention study

Supplementary Information The online version contains supplementary material available at https:// doi.org/10.1007/s13300-021-01108-9.

Members of the "AMD-OSDI Study Group and Nefrocenter Research and Nyx Start-Up Study Group" are listed in Acknowledgement section.

S. Gentile · G. Guarino · T. Della Corte · E. Satta .

M. Pasquarella · C. Romano · C. Alfrone

Nefrocenter Research and Nyx Start-Up, Naples, Italy

S. Gentile · G. Guarino · T. Della Corte · G. Marino Department of Internal Medicine, Campania

University "Luigi Vanvitelli", Naples, Italy

E. Satta

Polispecialistic Nephrological Center CNP Srl, Fratta Maggiore, Italy

L. Giordano

Emodialysis Center Srl, Nefrocenter Network,

Naples, Italy registered as the ISTERP-1 study. A total of 158 subjects with T2DM from the well-trained group of the 6-month-long ISTERP-1 study, all of whom had successfully attained lower glucose levels compared to baseline levels with lower daily insulin doses and with less frequent and severe hypoglycemic episodes, participated in the present investigation involving an additional 6-month follow-up period, called the ISTERP-2 study.

Methods: Participants were randomized into an intervention group and a control group, depending on whether they were provided or not provided with further education refresher courses for 6 months. At the end of the 6 months, the two groups were compared in terms of injection habits, daily insulin dose requirement, number of severe or symptomatic hypoglycemic events, and glycated hemoglobin (HbA1c) levels.

\section{F. Loiacono}

Nefrocenter Research, Naples, Italy

M. Capace

Vomero Center Crisci Bersabea \& C SNC,

Nefrocenter Network, Naples, Italy

R. Lamberti

Metelliano Medical Center, Nefrocenter Network, Naples, Italy

F. Strollo $(\bowtie)$

Endocrinology and Diabetes, IRCCS San Raffaele

Pisana, Rome, Italy

e-mail: felix.strollo@gmail.com 
Results: Despite being virtually superimposable at baseline, the two groups behaved quite differently during the follow-up. The within-group analysis of observed parameters showed that the subjects in the intervention group maintained and even improved the good behavioral results learned during the ISTERP-1 study by further reducing both the rate of injection technique errors $(p<0.001)$ and size of lipohypertrophic lesions at injection sites $(p<0.02)$. Conversely, those in the control group progressively abandoned best practice, except for the use of icecold insulin and, consequently, had significantly higher HbA1c levels and daily insulin dose requirements at the end of the follow-up than at baseline $(p<0.05)$. In addition, as expected from all the above, the rate of hypoglycemic episodes also decreased in the intervention group $(p<0.05)$, resulting in a significant difference between groups after 6 months $(p<0.02)$.

Conclusion: Our data provide evidence that intensive, structured education refresher courses have no outstanding durability, so that repeated refresher courses, at least at 6-month intervals, are needed to have positive effects on people with T2DM, contributing not only to prevention but also to long-term rehabilitation. Trial Registration: Trial Registration no. 118 bis/15.04.2018.

Keywords: Type 2 diabetes; Injection technique; Education; Rehabilitation; Lipohypertrophy; Hypoglycemia

\section{Key Summary Points}

A structured education program devoted to best insulin injection techniques (IT) improves metabolic control, exposes the individual to less frequent and less severe hypoglycemic events, and lowers daily insulin dose requirement.

The 6-month durability of such effects has not yet been the object of any assessment.
Contrary to indiviiduals with type 2 diabetes mellitus who were left unsupported, well-trained individuals who recieved intensive IT-specific educational support during another follow-up period maintained the excellent results attained after the first 6-month educational intervention.

This result calls for the systematic organization of periodic refresher courses as the effects of a structured, IT-oriented education-based rehabilitation program lasts $<6$ months.

The lack of such support at least partially explains the high rate of lipohypertrophy and related severe metabolic and economic consequences.

Researchers and scientific societies should strive for better institutional recognition and remuneration of structured educational programs as an undeferrable behavioral rehabilitation tool by pushing for and establishing such programs where absent.

\section{INTRODUCTION}

For persons with type 2 diabetes mellitus (T2DM), structured education plays a prominent role in injection technique improvement to avoid skin complications, including lipohypertrophy (LH), local inflammation, bruising, and subsequent, repeated unexplained hypoglycemic episodes (hypos). Significant changes in the absorption, pharmacokinetics, and pharmacodynamics of insulin can occur when insulin is injected into lipohypertrophic nodules, potentially leading to high glycated hemoglobin (HbA1c)) levels, glycemic variability, and changes in the daily insulin dose requirement [1-7]. Despite the risk factors for LH being very well known [6-8] and the publication of several papers on the rate of $\mathrm{LH}$ in different populations/care settings during the 
last few years [8-10], too little attention has been paid to LH prevention through education [11-14]. Even the few papers dealing with education on best insulin injection techniques suffer limitations as they are based on data from small case series or short-term observations. We recently published a case-control study using an intensive, structured education-based rehabilitation protocol with repeated refresher courses, which we refer to here as the "ISTERP1 " study [14]. However, even this study could not provide a clear-cut answer to the central question of whether or not single education cycles guarantee behavioral changes lasting $>$ 6 months, thus not resolving the issue of whether such education sessions represent a valuable, long-term behavioral rehabilitation tool.

Based on this background, the aim of the present investigation, named the ISTERP-2 study, was to assess the durability of structured education effects by monitoring for a further 6 months the injection habits and metabolic outcomes of well-trained participants in the ISTERP-1 study randomly divided into an intervention group (provided with further structured education refresher courses) and a control group (not receiving further follow-up refresher courses).

\section{METHODS}

This study was meant to be an extension of the recently published ISTERP-1 study [14] and was designed to be a two-arm, open-label, multicenter, randomized, case-control study. It was carried out in compliance with good clinical practice standards and in accordance to the ethical guidelines of the 1964 Declaration of Helsinki and its subsequent amendments. It was approved as a joint protocol by the Ethical and Scientific Committee of the reference center (University "Luigi Vanvitelli" Naples, Italy; trial registration no. 118 bis/15.04.2018), which served as the central reference ethical committee for all of the participating diabetes centers, with the latter an integral part of the same private consortium associated to the above-mentioned University, and by the Institutional Review Board (IRB Min. no. 9926, dated
05.02.2018). Before enrollment, all of the subjects with T2DM participating in the intensive education arm of the ISTERP-1 study signed an informed consent form to be included in the present investigation (see Fig. 1; Electronic Supplementary Material ISTERP-1 study), except for one who declined the invitation due to work constraints.

The primary endpoint was the ability to maintain correct insulin injection habits in relation to attending or not attending educational refresher programs. Secondary endpoints were: (1) metabolic control as reflected by HbA1c levels, (2) size of lipohypertrophic lesions, (3) daily insulin dose requirement and (4) rate of hypos.

All centers participating in the present study were part of the Nefrocenter Research Network in southern Italy-a private consortium supported by the National Health System under a special agreement with Naples University "Luigi Vanvitelli" relating to several clinical aspects, including the ethics committee. All participating centers used the same electronic record system, diagnostic/therapeutic procedures, and operating standards by adhering to the national program for continuous quality improvement. In addition, each center had their own healthcare professionals (HCPs) who had received specific training and were able to follow the study procedures appropriately.

Inclusion criteria were: having participated in the intervention group of the ISTERP-1 study for 6 months and thereby receiving two intensive courses interspersed with four phone-based refresher calls; being on a therapeutic regimen of at least three daily injections; and agreeing to perform self-monitoring of blood glucose (SMBG) systematically.

Exclusion criteria were: (1) any add-on hypoglycemic agents; (2) severe liver disease or cancer; (3) dementia or any other functional impairment affecting adherence to the study protocol; (4) participation in any other clinical trial; and (5) any-cause inability to attend all sessions regularly.

Once enrolled in the study, patients were then randomized to an intervention group and a control group according to a simple 


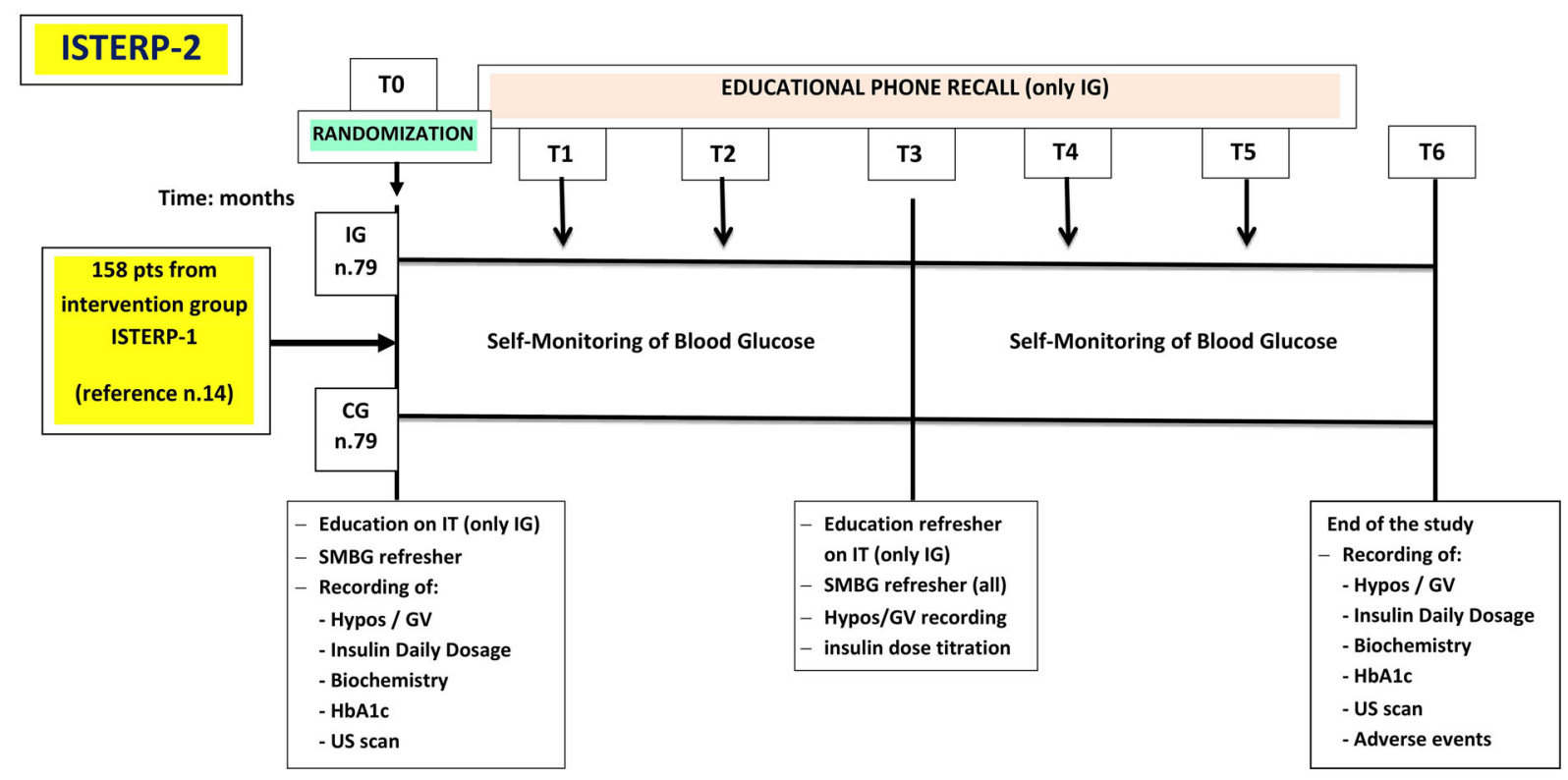

Fig. 1 Schematic representation of the study protocol. All 158 randomized patients had participated in the ISTERP1 and had received the educational training in that study according to exactly the same protocol that was provided to the intervention group in the present study. $C G$

centralized randomization system with blinding ensured by the envelope method.

Diagnostic criteria for T2DM and related complications/conditions, education material, equipment and methods used to perform SMBG monitoring, and the definition of and recording methods for severe hypoglycemia (SeHs) or symptomatic hypoglycemia (SyHs) were extensively described in our previous paper [14].

\section{Study Protocol}

Only patients from the previous study's intervention group entered the present follow-up study. They were evenly divided into the new intervention group $(n=79)$ and the new control group $(n=79)$ through an automatic random number generator. The intervention group benefited from a structured education session at 0 (i.e., baseline [T0]) and 3 months $(\mathrm{T}+3)$ with a monthly phone reminder at $1,2,4$, and 5 months $(\mathrm{T}+1, \mathrm{~T}+2, \mathrm{~T}+4$, and $\mathrm{T}+5$, respectively); the control group underwent no refresher courses and did not receive monthly

Control group, $G V$ glycemic variability, $H b A l c$ glycated hemoglobin, hypos hypoglycemic episodes, $I G$ intervention group, pts patients, $S M B G$ self-monitoring of blood glucose, T0 baseline, T1-T6 follow-up time points, US ultrasound

phone reminders until the 6 month time point $(\mathrm{T}+6)$.

In more detail, each injection technique education-based rehabilitation session involved ten people at most and lasted approximately 60 min. Each session made use of the BD Educational Starter Kit (Becton Dickinson, Inc., Franklin Lakes, NJ, USA), including site rotation grids, educational injection technique leaflets, and a blood glucose logbook. The LH "look and feel" teaching method used a BD Lipobox to provide visual and tactile clues for typical $\mathrm{LH}$ lesion identification. Patients were also instructed to rotate injection sites correctly and to avoid reusing needles to prevent the generation or worsening of LH. In addition, they received a leaflet with bullet points highlighting the role of correct injection habits in optimizing glucose control by keeping insulin pharmacokinetics and pharmacodynamics unaltered $[7,14]$. The complete sessions, eventually integrated by face-to-face in-depth analyses when needed, were performed as previously described [14] according to the Forum for Injection Technique (FIT) [15]. 
Clinical LH identification criteria are reported elsewhere $[6,8,14,16]$. Procedures used for defining injection habits, through a structured questionnaire, and SMBG-based hypo occurrence and symptom severity identification have been described in detail for the ISTERP-1 study [14]. Adherence to the study protocol was defined as good when the subject provided $>80 \%$ of expected recordings.

In addition, at $\mathrm{T} 0, \mathrm{~T}+3$, and $\mathrm{T}+6$, all patients underwent a complete visit, including a careful examinination of the injection site, and completed a self-administered questionnaire based on a salient, nonintrusive, recent-past-oriented, well-established procedure developed as part of the original Worldwide Injection Technique Questionnaire Study 2016 [7, 14, 16-18]. Highfrequency B-mode ultrasound skin scans were performed using the linear $20 \mathrm{MHz}$ probe (HD3; Philips NV, Amsterdam, The Netherlands) at all injection sites, as previously described [8], to compare palpatory/pinching impressions with objective findings and to define LH features appropriately. Two different blinded operators scanned the same patient. A $100 \%$ consistency in LH identification was preliminarily found among specialists regarding intra-operator, inter-operator and day-to-day operator variation independently of location, volume, extension, texture, or thickness [19].

This detailed procedure enabled investigators to add data to the electronic case report form on each patient at 3-month intervals, including detailed information on injection habits and hypo frequency or severity together with data on general biochemistry, HbA1c, daily insulin dose requirement, LH size, and eventually treatment-related adverse events (Fig. 1).

\section{Statistical Analysis}

In our previous educational intervention study [14], HbA1c levels decreased by $20 \%$ from baseline to the end of follow-up, with a standard deviation (SD) of $0.87 \%$. Based on these values, when setting the significance level at $\alpha=0.05$ (two-sided) and the power at $80 \%$, we calculated 60 patients as the minimum sample size for each group. When allowing for a $10-15 \%$ dropout rate, the required sample size increased to 75 cases per group. However, we included 79 cases per group, i.e., all the 158 subjects participating in the intervention group of our previous study who met the inclusion criteria for the present one.

Patient characteristics were reported as the mean \pm SD for continuous variables and as the number/percentage for categorical variables. SyHs and SeHs were expressed as incidence rates within 95\% confidence intervals (95\% CI) and were evaluated using the Poisson regression model. The significance of differences found between experimental treatments and times was tested using the repeated measures analysis of variance supplemented by the two-tailed paired Student's $t$ test with 95\% CIs for parametric variables and Mann-Whitney's $U$ test for nonparametric variables. The chi-square $\left(\chi^{2}\right)$ test with Yates's correction or Fisher's exact test was implemented for categorical variable differentiation. A $p<0.05$ was considered to be statistically significant. All evaluations were performed using the SAS release 9.4 statistical software program (SAS Institute, Cary, NC, USA).

\section{RESULTS}

All participants completed the study without reporting any treatment/device-related adverse effects. Median adherence to SMBG recording was as high as 90\% (range 87-100\%), and adherence to recording hypos was also good.

As shown in Table 1, baseline data for general and clinical parameters, including HbA1c, were statistically superimposable. Values in Table 2 show changes in items associated with the single injection technique in both groups and clearly depict different behaviors over time. Subjects in the intervention group achieved a further slightly but still significantly improved performance, with a 2 - to 3.8 -fold decrease in faulty behavioral elements $(p<0.001)$, and entirely avoided ice-cold insulin injection. Conversely, subjects in the control group performed progressively worse over the 6-month follow-up period $(p<0.001)$, with some 
Table 1 Main parameters for subjects randomly enrolled into the control group and intervention group

\begin{tabular}{lll}
\hline Baseline parameters & $\begin{array}{l}\text { Control } \\
\text { group } \\
\boldsymbol{n}=\mathbf{7 9}\end{array}$ & $\begin{array}{l}\text { Intervention } \\
\text { group } \boldsymbol{n}=\mathbf{7 9}\end{array}$ \\
\hline Male gender, $n(\%)$ & $70(41.36)$ & $72(39.48)$ \\
Age (years) & $64 \pm 11$ & $62 \pm 13$ \\
BMI (kg/m $\left.{ }^{2}\right)$ & $30.4 \pm 8.2$ & $30.5 \pm 7.7$ \\
HbA1c (\%) & $6.7 \pm 0.8$ & $6.5 \pm 01.0$ \\
DM duration (years) & $11.3 \pm 5.7$ & $11.6 \pm 9.8$ \\
$\begin{array}{l}\text { Injections/day (n) } \\
\text { Insulin treatment }\end{array}$ & $4.2 \pm 7.8$ & $6.9 \pm 8.3$ \\
$\quad$ duration (years) & & $58 \pm 13$ \\
Daily Insulin Dose & $56 \pm 12$ & \\
requirement (IU/day) & & \\
\hline
\end{tabular}

Values are presented as the mean \pm standard deviation (SD) unless indicated otherwise

$B M I$ Body mass index, $D M$ diabetes mellitus, HbAIc glycated hemoglobin

experiencing, for example, a $15 \%$ increase in daily insulin dose requirement $(46 \pm 9$ vs. $53 \pm 12$ IU, respectively; $p<0.044$ ) versus the roughly $7 \%$ decrease observed in the intervention group ( $46 \pm 9$ vs. $43 \pm 9 \mathrm{IU}$, respectively; $p<0.05)$, and a pronounced increase in the rate of SeHs and SyHs (ninefold $[p<0.00134]$ and fourfold [ $p<0.0198]$, respectively) as opposed to the dramatic drop observed within the intervention group $(p<0.05)$. Moreover, the cumulative hypo rate was significantly different between groups at the end of the follow-up $(p<0.00192)$, with SeHs being more frequent at night (60\% of the total) (Table 3). HbA1c levels stayed consistently low in the intervention group $(6.5+0.9 \%$ vs. baseline; $p=$ not significant) while worsening in the control group $(7.9+0.6$ vs. $6.6+0.8 \% ; p<0.05)$.

Interestingly, the control group maintained good performance in terms of ice-cold insulin injection, with the exception of three subjects.

Different injection habits most likely accounted for differences observed in the size of lipohypertrophic lesions between groups as measured using a structured, ultrasound-validated method [19] (Table 4). LH areas arbitrarily classified according to their diameter (i.e., $\geq 3$ or $<3 \mathrm{~cm}$ ) tended to distribute differently over time. At the end of the follow-up, the size of larger lipohypertrophic lesions decreased in $72.00 \%$ of cases in the intervention group compared to $41.37 \%$ in the control group $(p<0.0121)$, and smaller lipohypertrophic lesions remained the same size in $5.55 \%$ of cases in the intervention group compared to $22.00 \%$ of cases in the control group ( $p<0.00118)$, or even disappeared in $66.66 \%$ of cases vs. $38.99 \%$, respectively $(p<0.0119)$.

\section{DISCUSSION}

The authors of this follow-up study consider it to be a natural continuation of the ISTERP-1 study [14], with the aim to assess the durability of an intensive, structured education-based rehabilitation protocol. Indeed, the ISTERP-1 study suggested the hypothesis that people tend to forget most lessons learned and revert to bad habits as early as 6 months after attending a single structured training course, leading to worsening metabolic control and higher rates of hypoglycemic episodes. Conversely, a continuing 6-month education program resulted in maintained adherence to best injection techniques and reduced related healthcare costs by decreasing the frequency of hypoglycemic events while improving overall metabolic control, However, the scientific literature does not provide any information to date on either the durability of educational interventions or the best interval between refresher courses for sustained behavioral benefits.

As shown in Fig. 1, to fill this gap, we designed the ISTERP-2 study by randomizing the ISTERP-1 study's intervention group into two arms, with one group attending two 3-monthly refresher courses and receiving four interspersed educational phone recalls during a further 6-month period, and the other group not.

Our data provide clear evidence of progressively decreasing adherence to best injection practice by subjects in the control group who, 


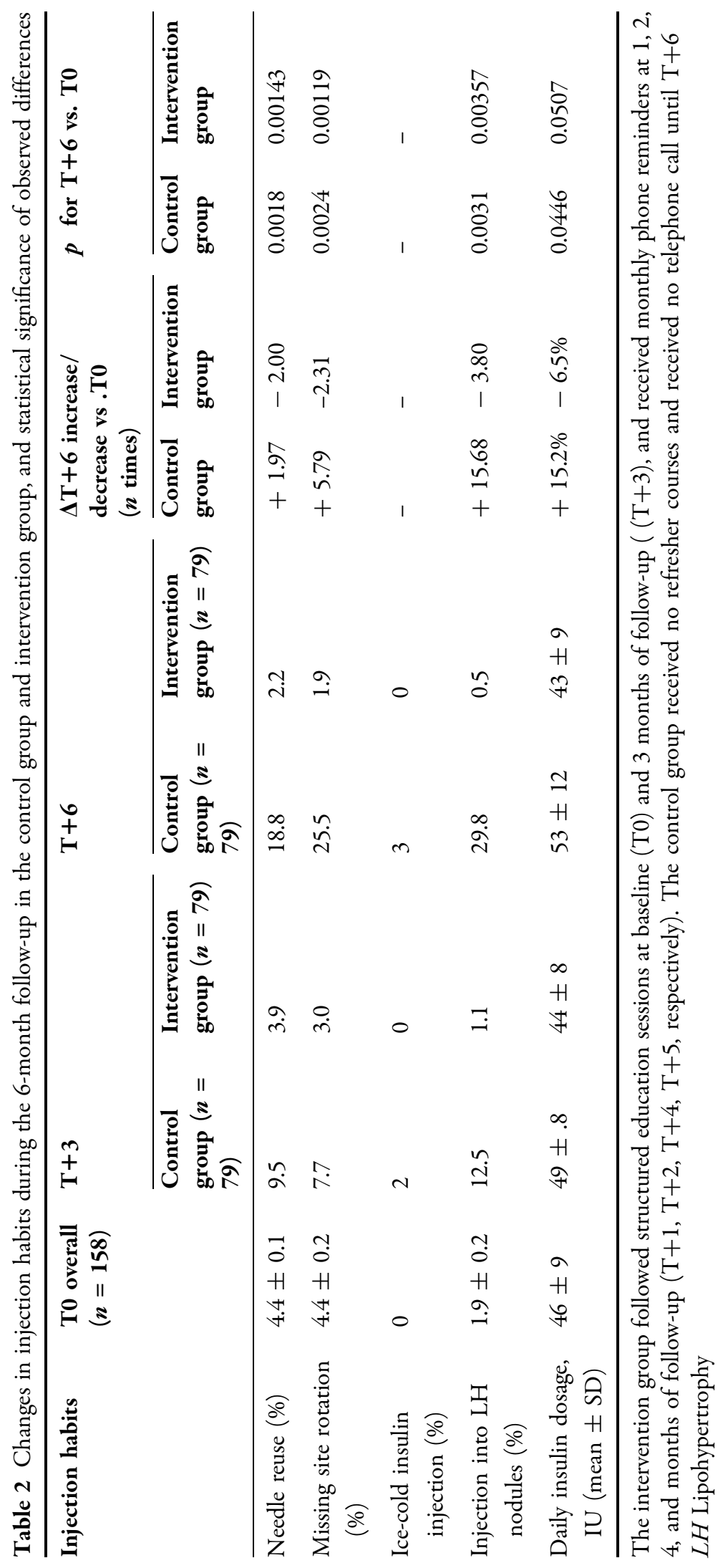


Table 3 Frequency of severe and symptomatic hypoglycemic events in the control group and the intervention group during the 6-month follow-up, and significance of observed differences

\begin{tabular}{|c|c|c|c|c|c|c|}
\hline Study groups & Hypoglycemic episodes & $\begin{array}{l}\text { T0 }(n= \\
158 \\
\text { overall })\end{array}$ & $\begin{array}{l}\text { T+3 }(n= \\
79)\end{array}$ & $\begin{array}{l}T+6 \\
(n=79)\end{array}$ & $\begin{array}{l}\Delta \mathrm{T}+6 \text { increase } / \\
\text { decrease vs. T0 } \\
(n \text { times })\end{array}$ & $p$ \\
\hline \multirow{3}{*}{$\begin{array}{l}\text { Control group } \\
(n=79)\end{array}$} & Severe & $1(1.26)$ & $5(6.32)^{*}$ & $9(11.39)$ & +9.0 & 0.00134 \\
\hline & Symptomatic & $6(7.59)$ & $\begin{array}{l}14 \\
\quad(17.72)^{* *}\end{array}$ & $\begin{array}{l}24 \\
\quad(30.37)\end{array}$ & +4.0 & 0.0198 \\
\hline & Overall & $8(10.12)$ & $\begin{array}{l}19 \\
(24.05)^{* *}\end{array}$ & $\begin{array}{l}33 \\
\quad(41.77)\end{array}$ & +4.12 & 0.0189 \\
\hline \multirow{4}{*}{$\begin{array}{l}\text { Intervention } \\
\text { Group }(n= \\
79)\end{array}$} & Severe & 0 & 0 & 0 & - & - \\
\hline & Symptomatic & $6(7.59)$ & $2(2.53)^{*}$ & $3(3.79)$ & -0.5 & 0.05 \\
\hline & Overall & $6(7.59)$ & $2(2.53)^{*}$ & $3(3.79)$ & -0.5 & 0.05 \\
\hline & $\begin{array}{l}\text { Symptomatic control group } \\
\text { vs. intervention group }\end{array}$ & n.s & 0.00128 & 0.00192 & & \\
\hline
\end{tabular}

Values are presented as the number with the percentage in parentheses

${ }^{*},{ }^{* *}$ Significant at ${ }^{*} p \leq 0.05$ and ${ }^{* *} p<0.001$ vs. baseline (T0)

Table 4 Changes in the diameter of larger lipohypertrophic lesions in the control group and intervention group during the 6-month follow-up and significance of observed differences

\begin{tabular}{llllll}
\hline Study group & LH diameter $(\mathbf{c m})$ & Baseline & \multicolumn{2}{l}{ End of follow-up } \\
\cline { 4 - 6 } & & & Undetectable & Reduced & Unchanged \\
\hline Intervention group $(\mathrm{IG})(n=79)$ & $>3$ & $25(31.64)$ & $2(8.0)$ & $18(72.00)$ & $5(20.00)$ \\
& $<3$ & $54(68.35)$ & $36(66.66)$ & $15(27.77)$ & $3(5.55)$ \\
Control group $(\mathrm{CG})(n=79)$ & $>3$ & $29(36.70)$ & $1(3.44)$ & $12(41.37)$ & $16(55.17)$ \\
& $<3$ & $50(63.29)$ & $19(38.00)$ & $20(40.00)$ & $11(22.00)$ \\
& IG vs. CG $(\geq 3 \mathrm{~cm})$ & n.s & n.s & $<0.0121$ & $<0.00246$ \\
& IG vs. CG $(\leq 3 \mathrm{~cm})$ & n.s & $<0.0119$ & $<0.0518$ & $<0.00118$ \\
\hline
\end{tabular}

Values in table are presented as a number with the percentage given in parentheses

In both groups, LH size significantly changed: $80 \%$ (IG) vs. $45 \%$ (CG) ( $p<0.001)$ for LH $>3 \mathrm{~cm}$, and $71 \%$ (IG) vs. $35 \%$ (CG) $(p<0.01)$ for smaller lesions. LH persistence was lower in the IG than in the CG $(10.7$ vs. $34.175 \%$, respectively; $p<0.01)$

despite previous intensive training, showed incorrect practices in terms of needle reuse (twice the baseline level), missing site rotation (sixfold higher than baseline rate), and intra-LH injection (15-fold higher than baseline rate), with a consequent $15.2 \%$ increase in daily insulin dose requirement. The only good practice retained by both groups was avoiding icecold insulin utilization. 
Within the context of optimal post-ISTERP-1 study baseline conditions, the most relevant clinical consequences were the higher hypo rates and HbA1c levels found in the control group compared to the intervention group, and the decreased LH size observed in the latter only. Such findings provide indirect evidence that subjects in the control group resumed injecting insulin into LH lesions during the education-free period.

Our data show, for the first time, that continuing education plays an essential role in maintaining correct injection practice beyond 6 months. Some authors of relatively small case series involving different 3- to 6-month education interventions have suggested improved performance after training [11-13, 19, 20]. However, to the best of our knowledge, no data are available on the effects of structured education programs lasting longer than 6 months. We note that the length of the combined ISTERP-1 and ISTERP-2 studies is 12 months and that our results show that even well-trained and high-performing subjects tend to slip back into bad injection habits quite quickly when message-strengthening procedures are missing.

Indeed, it is challenging to maintain correct injection habits all the time. Unfortunately, we have no way at this time to identify the factors underlying "lost adherence" and can only hypothesize that longstanding bad habits are hard to get rid of as a result of a mix of painless nature of injections into partially denervated LH areas [8], laziness, depression, lack of interest in personal health conditions, and automatism involving involuntary repetition of acquired movements. To the contrary, it is common knowledge that behavioral changes do not stay active for very long despite being attained through sustained efforts.

\section{Limitations}

The main limitation of our study is the relatively small number of people involved that, however, is justified by the considerable effort required by the intensive, multimodal, structured education protocol adopted.

\section{CONCLUSIONS}

The present investigation represents the natural continuation of the ISTERP-1 study by extending to 12 months the observation periods of T2DM patients consistently attending an intensive, multimodal education-based rehabilitation program aimed at best injection practice. Subjects randomized to the control group were left unattended for an additional 6 months during the ISTERP-2 study after undergoing the abovementioned 6-month program during the ISTERP-1 study. It was noted that these subjects at least partially returned to bad injection habits, resulting in worsening metabolic control despite increasing daily insulin doses, thus showing that the lack of continuing educational support rapidly thwarts the considerable efforts made by healthcare teams to provide T2DM patients with intensive 3-monthly refresher courses.

Our results provide evidence that insulintreated T2DM patients need intensive educational refresher courses minimally at 3-month intervals. Furthermore, based on the lessons learned from these studies, clinicians should pay much more attention to structured therapeutic education on best injection practices than they do now. By providing more education, HCPs could provide people suffering from a potentially disabling chronic disease with a valuable rehabilitation tool based on the systematic prevention of LHs and related complications $[8,9]$.

These data indirectly explain why LHs are so frequent: according to the current literature, LHs affect $38 \%$ insulin-treated patients on average [10] and more than $60 \%$ of them in many cases [14]. Indeed, it must be admitted that healthcare systems worldwide are still characterized by their utmost attention to best drug regimen choices with total oblivion of systematic identification of skin lesions and suitable LH-prevention education [7].

\section{Final Remarks}

We could provide evidence that (1) well-trained T2DM patients lose at least part of their health 
benefits within 6 months of clinically adequate yet education-free medical assistance and (2) continuing education involving an intensive, multimodal structured intervention is crucial to achieving a persistent change in individual behavior. Efforts to provide continuing education have the invaluable merit of providing people with T2DM with positive effects on wellestablished disease parameters and thus contributing to complication prevention and longterm rehabilitation.

Therefore, we firmly believe that policymakers, scientific societies, and academic institutions organizing under-graduate and postgraduate courses should promote concrete actions to enhance and financially support the role of education by adequate resource allocation and formal professional recognition of diabetes educators. This choice would undoubtedly be cost-effective by lowering direct and indirect healthcare costs and, as documented by our previous paper on the ISTERP-1 study [14], by improving the quality of life of well-trained patients.

\section{ACKNOWLEDGEMENTS}

We extend our sincerest thanks to the doctors and nurses of the participating centers. We are also very grateful to the patients for their invaluable and generous participation in the study, as well as for providing their enthusiastic consent to the publication of results arising from the dataset they had contributed to. We also feel deeply indebted to Becton Dickinson Italia S.p.A. (Milan, Italy) for providing educational material with no conditions attached. We are also indebted to all experimenters and components of the AMD-OSDI study group and of the Nefrocenter working group, all of whom are listed in Reference [14], al DCs were part of the Nefrocenter Research Network in Southern Italy, a private consortium supported by theNational Health System in association with Naples University "Luigi Vanvitelli" for several clinical aspects, including theethics committee2.
Members of the AMD-OSDI Study Group: Stefano De-Riu, Nicoletta De-Rosa, Giorgio Grassi, Gabriella Garrapa, Laura Tonutti, Katija Speese, Lia Cucco, MariaTeresa Branca, Amodio Botta.

Members of the Nefrocenter Research and Nyx Start-Up Study Group: Diabetologists: Sandro Gentile, Giuseppina Guarino, Felice Strollo, Gerardo Corigliano, Marco Corigliano, Carmine Martino, Antonio Fasolino, Antonio Vetrano, Agostino Vecchiato, Domenica Oliva, Clelia Lamberti, Domenico Cozzolino, Clementina Brancario, Luca Franco. Nutritionist: Teresa Della-Corte. Nephrologists: Carmelo Alfarone, Maria Luisa Abate Giovanna, Maria Amicone, Giovanni Apuzzo, Gennaro Barbuto, Antonio Bassi, Pasquale Boccia, Francesca Borghesi, Alfonso Bosco, Francesco Buono, Tiziana Castellano, Giorgio Chianese, Michele Cicala, Alfonso Ciotta, Secondino Cipriano, Fabrizio G. Crisci, Cristiano Pina, Iris Cupic, Marco De Chiara, Alfonso De Maio, Carlo Del Piano, Chiara Del Prete, Luigi Di Leva, Monica Di Maio, Mauro Di Monte, Alfonso Donnarumma, Enzo Di Stazio, Michele Fabozzi, Maria Gallo, Laura Giordano, Monte Giovanni, Manuela Guerri, Giulia Esposito Iacobitti, Anna Maria La Manna, Gianluca Latte, Fabrizio Lo Iacono, Donato Maietta, Chiara Marano, Maria Federica Finelli, Maria Pasquarella, Mario Acquaviva, Massimo Romano, Gennaro Mattiello, Pietro Miano, Silvia Migliaccio, Alfredo Mignone, Giovanni Monte, Alfredo Fabio Murano, Simona Oliviero, Teresa Pagano, Gabriele Palmentola, Salvatore Postiglione, Pvalo Yavorskiy, Raffaela Esposito, Rosario Reggio, Bruno Riccardi, Eleonora Riccio, Giuseppe Romano, Ersilia Satta, Francesco Antonio Savino, Luisa Scarpati, Domenico Schettino, Giuseppe Spinoso, Erika Troncone, Pasquale Vendemia, Olga Yushkova. Nurses: Paolino Albertini, Lucio Ambrosino, Rosa Vitale Amoroso, Marilena Angrisani, Rosa Apuzzo, Angela Auletta, Fabrizio Barbaro, Gilda Barrella, Alfonso Bartiromo, Maria Battipaglia, Orfeo Belardo, Roberto Bernardo, Valentina Bianco, Pasquale Biondillo, Lucia Bottiglieri, Michela Brida, Orsola Brusco, Giuseppina Buonocore, Zaira Buonocore, Halina Buska, Giuseppe Calabrese, Ida Campolattano, Margherita Capasso, 
Cecilia Caracciolo, Teresa Carrara, Angela Casaburo, Sara Caso, Marina Assunta Cesarano, Tiziana Cesarini, Annunziata Cherillo, Enrico Cicchella, Michelina Cicchella, Angelo Cimmarosa, Simone Cimmino, Carmelina Cirillo, Pasquale Como, Tiziana Conturso, Giuseppina Cozzolino, Adele Crispino, Raffaele D'Angelo, Salvatore De Felice, Adriano De Filippis, Margarita De Lucia, Lucia De Micco, Maria Andrea De Vita, Antonio Decostanzo, Carmine D'Elia, Salvatore De Felice, Eligio Della Monica, Angela D'Errico, Veronica D'Esculapio, Marialucia Di Riso, Giovanna Di Maio, Roberta Di Maio, Assunta Di Matola, Assunta Di Nardo, Elisabetta Di Virgilio, Davide Doriano, Eliana Ebraico, Gioacchino Erbaggio, Luca Erpete, Roberta Errichiello, Santo Esca, Gennaro Esposito, Virginia Esposito, Rinaldo Fargnoli, Pasquale Ferrante, Oriana Ferraro, Marco Festinese, Carmen Figlioli, Giovanni Fiorenza, Filomena Fontanella, Michela Fusco, Carmela Gigante, Carmelina Giove, Ruslava Gladka, Anna Guerra, Achille Iannone, Lucia Imbembo, Concetta Imbimbo, Melania Imbimbo, Grazia Indaco, Felice Marco Isola, Antonietta Izzo, Beata Jeschke, Onorina Letizia, Danilo Lettieri, Ana Maria Mandato, Donatella Mannato, Erika Manzi, Lucia Manzo, Carmela Marano, Zuzanna Matusz, Emilio Menna, Sara Milano, Joanna Mlynarska Malgorzata, Carmela Montesarchio, Vincenza Morgillo, Vincenzo Morgillo, Teresa Morrone, Teresa Napolitano, Maria Teresa Natale, Aldo Occhio, Livio Orropesa, Daniela Palmeri, Angela Palmiero, Antonietta Pandolfo, Valentina Passa, Assunta Pastore, Teresa Jadwiga Pazdior, Annamaria Pellino, Elena Petrone, Valentina Pettinati, Filomena Piccolo, Catello Polichetti, Milena Puce, Angela Rainone, Emanuela Repola, Raffaele Riccio, Amelia Ricuperati, Maria Roselli, Enza Ruotolo, Eva Russo, Francesco Russo, Antonietta Salsano, Andrea Schettino, Annalisa Siani, Marilina Siani, Immacolata Silvestri, Pellegrino Spallieri, Annunziata Stasio, Claudia Tabacco, Francesca Tammaro, Maria Emanuela Toscano, Gabriele Ummarino, Federica Variselli, Francesca Vela, Mario Vitale, Paolo Vitale.

Funding. No funding or sponsorship was received for this study or the publication of this article. No payment was requested for publication and online posting costs. None of the authors or coworkers received funding or another type of payment for this paper.

Editorial and Other Assistance. Special thanks are due to Dr. Paola Murano of Nefrocenter Research for providing continuous complimentary logistical support, and to members of AMD-OSDI Study Group on Injection Technique for their editorial assistance and critical manuscript revision.

Authorship. All named authors (Sandro Gentile, Giuseppina Guarino, Teresa Della Corte, Giampiero Marino, Ersilia Satta, Carmine Romano, Carmelo Alfarone, Maria Pasquarella, Laura Giordano, Fabrizio Loiacono, Maurizio Capece, Rossella Lamberti and Felice Strollo) meet the International Committee of Medical Journal Editors (ICMJE) criteria for authorship for this article, take responsibility for the integrity of the work as a whole, and gave their approval for this version to be published.

Authorship Contributions. SG and FS designed the study and wrote the article. ES, TDC, GG, GM, CR, CA, LG, FL, MC, RL and MP critically read and approved the paper. All authors complied data, critically assessed the results, and approved the final text. All collaborators critically read and approved the final text.

Disclosures. Sandro Gentile, Giuseppina Guarino, Teresa Della Corte, Giampiero Marino, Ersilia Satta, Carmine Romano, Carmelo Alfarone, Maria Pasquarella, Laura Giordano, Fabrizio Loiacono, Maurizio Capece, Rossella Lamberti and Felice Strollo have no financial interests to declare in relation to the present study.

Compliance with Ethics Guidelines. This study was carried out in compliance with good clinical practice standards and in accordance to the ethical guidelines of the 1964 Declaration of Helsinki and its subsequent amendments. It was approved as a joint protocol by the Ethical and Scientific Committee of the reference center 
(University "Luigi Vanvitelli" Naples, Italy; trial registration no. 118 bis/15.04.2018), which served as the central reference ethical committee for all of the participating diabetes centers, with the latter an integral part of the same private consortium associated to the above-mentioned University, and by the Institutional Review Board (IRB Min. no. 9926, dated 05.02.2018). Before enrollment, all of the subjects with T2DM participating in the intensive education arm of the ISTERP-1 study signed an informed consent form to be included in the present investigation (see Fig. 1; Electronic Supplementary Material ISTERP-1 study), except for one who declined the invitation due to work constraints.

Data Availability. The datasets analyzed during the present study are available from the corresponding author on reasonable request.

Open Access. This article is licensed under a Creative Commons Attribution-NonCommercial 4.0 International License, which permits any non-commercial use, sharing, adaptation, distribution and reproduction in any medium or format, as long as you give appropriate credit to the original author(s) and the source, provide a link to the Creative Commons licence, and indicate if changes were made. The images or other third party material in this article are included in the article's Creative Commons licence, unless indicated otherwise in a credit line to the material. If material is not included in the article's Creative Commons licence and your intended use is not permitted by statutory regulation or exceeds the permitted use, you will need to obtain permission directly from the copyright holder. To view a copy of this licence, visit http://creativecommons.org/licenses/by$\mathrm{nc} / 4.0 /$.

\section{REFERENCES}

1. Thow JC, Home PD. Insulin injection technique: depth of injection is important. Br Med J. 1990;301: 3-4.
2. Strauss K, De Gols H, Letondeur C, Matyjaszczyk M, Frid A. The second injection technique event (SITE), May 2000, Barcelona Spain. Pract Diabetes Int. 2002;19:17-21.

3. Nakatani Y, Matsumura M, Monden T, Aso Y, Nakamoto T. Improvement of glycemic control by re-education in insulin injection technique in patients with diabetes mellitus. Adv Ther. 2013;30(10):897-906. https://doi.org/10.1007/ s12325-013-0066-8.

4. Gentile S, Agrusta M, Guarino G, et al. Metabolic consequence of incorrect insulin administration techniques in aging subjects with diabetes. Acta Diabetol. 2011;48:121-5. https://doi.org/10.1007/ s00592-009-0172-x.

5. Famulla S, Hovelmann U, Fischer A, Coester HV, Hermanski L, Kaltheuner M. Insulin injection into lipohypertrophic tissue: blunted and more variable insulin absorption and action and impaired postprandial glucose control. Diabetes Care. 2016;39: $1486 \mathrm{e} 92$.

6. Strollo F, Guarino G, Armentano V, et al. Unexplained hypoglycaemia and large glycaemic variability: skin lipohypertrophy as a predictive sign. Diabetes Res Open J. 2016;2(1):24-32.

7. Blanco M, Hernandez MT, Strauss KW, Amaya M. Prevalence and risk factors of lipohypertrophy in insulin-injecting patients with diabetes. Diabetes Metab. 2013;39(5):445-53. https://doi.org/10.1016/ j.diabet.2013.05.006.

8. Gentile S, Guarino G, Corte TD, et al. Insulin-induced skin lipohypertrophy in type 2 diabetes: a multicenter regional survey in Southern Italy. Diabetes Ther. 2020;11(9):2001-17. https://doi.org/10. 1007/s13300-020-00876-0.

9. Gentile S, Strollo F, Guarino G, Diabetologists, Nurses. Why are so huge differences reported in the occurrence rate of skin lipohypertrophy? Does it depend on method defects or on lack of interest? Diabetes Metab Syndr. 2019;13(1):682-6. https:// doi.org/10.1016/j.dsx.2018.11.042.

10. Deng N, Zhang X, Zhao F, Wang Y, He H. Prevalence of lipohypertrophy in insulin-treated diabetes patients: a systematic review and meta-analysis. J Diabetes Investig. 2017;9(3):536-43. https://doi. org/10.1111/jdi.12742.

11. Grassi G, Scuntero P, Trepiccioni R, Marubb F, Strauss K. Optimizing insulin injection technique and its effect on blood glucose control. J Clin Transl Endocrinol. 2014;1(4):145-50.

12. Smith M, Clapham L, Strauss K. UK lipohypertrophy interventional study. Diabetes Res Clin Pract. 
2017;126:248-53. https://doi.org/10.1016/j.diabres. 2017.01.020.

13. Gentile S, Strollo F, Nefrocenter Research Study Group. Cost saving effects of a short-term educational intervention entailing lower hypoglycaemic event rates in people with type 1 diabetes and lipohypertrophy. Diabetes Res Clin Pract. 2018;143: 320-1. https://doi.org/10.1016/j.diabres.2018.07. 030.

14. Gentile S, Guarino G, Della Corte T, et al. Role of structured education in reducing lypodistrophy and its metabolic complications in insulin-treated people with type 2 diabetes: a randomized multicenter case-control study. Diabetes Ther. 2021. https://doi. org/10.1007/s13300-021-01006-0.

15. FIT UK Forum for Injection Technique UK. The UK injection and infusion technique recommendations, 4th ed. 2016. http://www.fit4diabetes.com/ files/4514/7946/3482/FIT_UK_Recommendations_ 4th_Edition.pdf. Accessed 12 May 2021.

16. Gentile S, Guarino G, Della Corte T, et al. Lipohypertrophy in elderly insulin-treated patients with type 2 diabetes. Diabetes Ther. 2020. https://doi. org/10.1007/s13300-020-00954-3.

17. Frid AH, Hirsch LJ, Menchior AR, Morel DR, Strauss KW. Worldwide injection technique questionnaire study: population parameters and injection practices. Mayo Clin Proc. 2016;91(9):1212-23. https:// doi.org/10.1016/j.mayocp.2016.06.011).

18. Frid AH, Hirsch LJ, Menchior AR, Morel DR, Strauss KW. Worldwide injection technique questionnaire study: injecting complications and the role of the professional. Mayo Clin Proc. 2016;91(9):1224-30. https://doi.org/10.1016/j.mayocp.2016.06.012 .

19. Frid AH, Kreugel G, Grassi G, et al. New insulin delivery recommendations. Mayo Clin Proc. 2016;91(9):1231-55. https://doi.org/10.1016/j. mayocp.2016.06.010.

20. Gentile S, Guarino G, Giancaterini A, Guida P, Strollo F, AMD-OSDI Italian Injection Technique Study Group. A suitable palpation technique allows to identify skin lipohypertrophic lesions in insulintreated people with diabetes. Springerplus. 2016;5(5):563. https://doi.org/10.1186/s40064-0161978-y.

21. Campinos C, Le Floch JP, Petit C, et al. An effective intervention for diabetic lipohypertrophy: results of a randomized, controlled, prospective multicenter study in France. Diabetes Technol Ther. 2017;19(11):623-32. https://doi.org/10.1089/dia. 2017.0165. 